\title{
Design of Hybrid Blind Detection Based Spectrum Sensing Technique
}

\author{
V. Sharma*, S. Joshi \\ Department of Electronics \& Communication Engineering, Maharana Pratap University of \\ Agriculture and Technology, CTAE, Udaipur, Rajasthan, India
}

Received 27 April 2020, accepted in final revised form 15 July 2020

\begin{abstract}
Cognitive Radio is a boon to efficient utilization of spectrum to meet the demand of next generation. Spectrum Sensing (SS) is an active research area, essential to meet the requirement of efficient spectrum utilization as it detects the vacant bands. This paper develops a Hybrid Blind Detection (HBD) technique for cooperative spectrum sensing which combines the Energy Detector (ED) and the Anti-Eigen Value Detection (AVD) techniques together to enhance the detection accuracy of a cognitive radio. Collaboration among the cognitive users is achieved to reduce the error and hard fusion based detection is implemented to detect the existence of primary user. The detection accuracy of the design is evaluated with respect to detection probabilities and the results are examined for improvements with the traditional two stage detection techniques. Fusion rules for the cooperative environment are implemented and compared to detect majority rule suitable for the proposed design.
\end{abstract}

Keywords: Cognitive radio; Blind detection; Energy detection; Fusion rule.

(c) 2020 JSR Publications. ISSN: 2070-0237 (Print); 2070-0245 (Online). All rights reserved. doi: http://dx.doi.org/10.3329/jsr.v12i4.46870 J. Sci. Res. 12 (4), 575-583 (2020)

\section{Introduction}

Cognitive radio systems normally contain licensed users (also known as primary users) of the spectrum, who are current license-holders and secondary users who look for using the spectrum as per the availability of vacant bands when the primary users are inactive. Due to the presence of cognitive radios the interference is increased and the quality-of-service of the primary user can be degraded. This increased interference for the PUs must be kept at a minimal level. It is required therefore, that cognitive radios sense and verify the spectrum availability and even weak primary user signals can be distinguished by them. Thus, spectrum sensing is considered as cognitive radio's most essential functionalities [1].

As we have discussed, spectrum sensing is considered as a task where various radio channel parameters are guesstimated, these parameters are power availability, spectrum availability, noise level, interference level, transmission channel characteristics, etc.

*Corresponding author: vatsalasharma01@gmail.com 
Mainly spectrum sensing is carried out in the domain of time and frequency. Although, phase as well as code domains can also be used for carrying out spectrum sensing [2].

For the PUs or licensed users being there, the CRs or unlicensed users have to incessantly observe the spectrum. If for a specific time, the PU is not present, then the licensed spectrum can be exploited by CR until PU comes back. The spectrum must be vacated by the Cognitive Radio (CR) after the primary User (PU) recurs and CR is moved to another unemployed spectrum. It is clear that the complete spectrum must be monitored continuously by the Cognitive Users (CRs/CUs) so that it won't miss any opportunity for using the channel which $\mathrm{PU}$ is not utilizing at that time. When the spectrum is continuously monitored, such scheme is known as Spectrum Sensing (SS). CRs' primary goal is the spectrum utilization which causes the sensing of the spectrum as critical and significant CR process. There exist two cases where unexploited spectrums are available, that are a spatial vacant spectrum or a temporal idle spectrum. A spatial idle spectrum occurs whilst a particular geographical region is used by the PU for transmission and outside that area spectrum is used by the CRs. A temporal vacant spectrum occurs while for a specific time period there is no transmission by the PU and for that period the spectrum is used by the CRs. Although, there exists various parameters that has effect on performance of spectrum sensing like multipath fading, shadowing and noise uncertainty. Mainly there are two categories of sensing techniques: Local spectrum sensing, where an autonomous/ self-governing decision is made by CR which concerns the PU's presence; secondly, the cooperative spectrum sensing, where PU's presence is decided by the group of CRs [3].

Yucek and Arslan [4] analyze various spectrum sensing schemes. Different features of the spectrum sensing was discussed. Various sensing methods were considered and collaborative sensing was proposed. Although, practical wireless phenomenon such as multipath shadowing and fading deteriorates the local SS [4]. Therefore, there was need of an active technique for improvement of detection performance which is proposed by Akyildiz et al. [5] in which he exploited the spatial diversity. Basically, such practical phenomenon must be considered for the detection performance and therefore, such effects are mitigated by the cooperative spectrum sensing [5]. Satheesh et al. [6] compared a standard energy detector to cyclostationary detector for a BPSK signal. Their results showed that cyclostationary detector has a higher probability of detection, especially at low SNR values, when compared to conventional energy detector [6]. Emara et al. [7] considered two SS techniques for cognitive radios; first is the energy based detection and second is based on multi taper frequency approximation scheme [7]. Energy based detector was analyzed for single user as well as multiple users collaborated using majority fusion rule also known as k out of $M$ hard decision based fusion rule. They verified results of both scenarios by genetic algorithm optimizer. Simulation results showed better performance of cooperative sensing in comparison of single user sensing. Zhai et al. [8] proposed a two stage spectrum sensing architecture in frequency domain that was based on SVM (Support Vector Machine). It shows that under regime of lower signal to noise ratio, the sensing accuracy can be improved to a large extend by using more than one 
sensing stages in a detector. Awin et al. [9] discussed blind Spectrum Sensing techniques for interlaced CR networks that included the detailed description of all the spectrum sensing techniques which does not need any information before the reception of the primary user signal. It also explains the comparative analysis of all the elaborated blind and semi blind spectrum sensing techniques.

Concluding the literature review of energy detection based spectrum sensing techniques till date, a Hybrid Blind Detection (HBD) based spectrum sensing technique is proposed to improve the detection accuracy of the cognitive user in terms of detection probabilities. As per our best knowledge, energy detection combined with and anti-Eigen value based detection has not been studied in literature till date. The following sections of the paper presents the system model of the proposed design with its mathematical modelling followed by the implementation and at last the simulation results are analyzed in terms of detection accuracy.

\section{System Model}

Spectrum Sensing (SS) is the dominant part of the cognition cycle in CRNs because it monitors the entire consecutive mechanism which is based on its outcome [10]. In the SS phase, a CR explores to recognize the temporarily unused spectrum frequencies (spectrum holes) as well as exploit them opportunistically without interfering with the PU. Lots of local spectrum sensing approaches have been used to achieve SS task. Spectrum detecting is a key capacity of subjective radio to anticipate the destructive obstruction with authorized clients and distinguish the accessible range for improving the range access. In any case, identification execution again and again is regularly undermined with multipath blurring, shadowing and collector vulnerability issues. The implementation of the spectrum sensing algorithms can be modeled as a binary hypothesis in order to evaluate the performance of the techniques. The hypothesis representation is as follows:

$$
\begin{aligned}
& \text { A0: } y(t)=n(t), \\
& \text { Al: } y(t)=h^{*} x(t)+n(t)
\end{aligned}
$$

Where received signal is represented with $y(t)$, the primary user signal is represented with $\mathrm{x}(\mathrm{t})$, additive white Gaussian noise is represented with $n(t)$ as well as the channel gain of the primary user is represented with $\mathrm{h}$. The hypothesis $A 0$ is a null hypothesis that implies that $\mathrm{PU}$ is absent while $A 1$ shows the existence of the primary signal [11].

The proposed model consists of two parallel blind detectors namely energy detector (ED) and Anti-Eigen value detector (AVD) acting for high SNR signal and low SNR signals respectively. The combined decision from each cognitive user is send to the fusion center (FC) to obtain the decision for the existence of primary user. The block diagram of the proposed system model is shown in Fig. 1. 


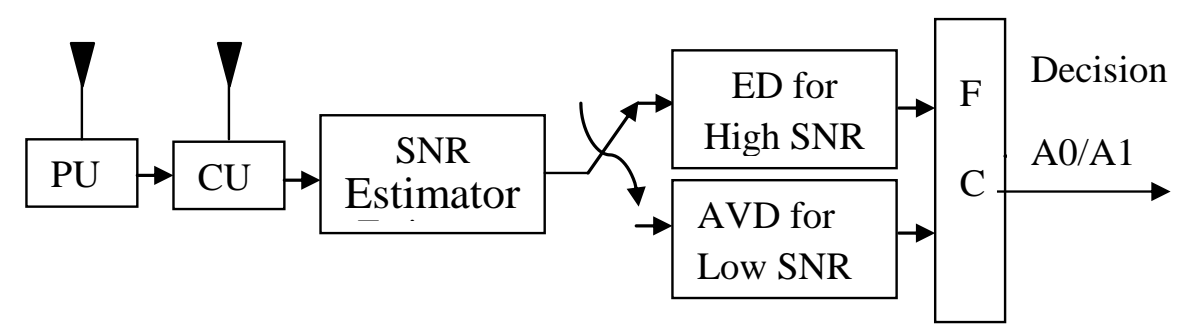

Fig. 1. Block diagram of HBD.

The signal received from the PU is received by the $\mathrm{CR}$ which calculates the signal to noise ratio first to decide the suitable detector for the sensing the signal as ED gives accurate results for high SNR but fails at low SNR regime. Therefore, a signal with high SNR is given to ED detector while a signal with low SNR is given to AVD detector respectively to sense the presence or absence of PU signal. The signal is received in the form of samples; the detection accuracy also depends on the number of samples taken by the detector to sense the signal. Then finally all the decisions are embed to the fusion center where the majority voting rule gives the result for the existence of the PU signal.

\section{Methodology Implemented}

The proposed two stage HBD algorithm implements two detectors simultaneously for low SNR values as well as high SNR values accordingly. A pre-set threshold based energy detector is utilized for the signal containing more SNR approximation and the signal having lesser SNR is detected by Anti-Eigen value detector on the basis of their advantages and implementation issues. In second stage, the fusion center employs approximated test statistics values of the received signal as input data to obtain the conclusion.

\subsection{Energy detector $(E D)$}

Under the assumption of constant features of the primary user in the sensing duration, each sample undergoes fading independently. Each antenna is equipped with enhanced energy detector which works on the following test statistics to detect the PU [12]:

$$
T_{i}=\sum_{j=1}^{k}\left(\frac{y_{i}(j)}{\sigma_{n}}\right)^{2}
$$

where $i=1$ to $u$ and $j=1$ to $k$. where $k$ denotes total number of samples and $\sigma_{\mathrm{n}}$ denotes the noise variance. $y_{\mathrm{i}}(j)$ is the received signal at CR. If test statistics $\mathrm{T}_{\mathrm{i}}$ is greater than the predefined threshold value $\gamma$, the output is 1 which represents the existence of the PU signal whereas if $T_{\mathrm{i}}$ less than the predefined threshold value $\gamma$, the output is 0 which denotes the absence of PU signal, resulting in immediate allotment of the vacant band to CR [13]. The value of predefined threshold is given by 


$$
\gamma=\frac{\sigma_{n}^{2}}{S N R}\left[1+\sqrt{2 / k} Q^{-1} P(f)\right]
$$

Where $k$ denotes the count of samples of the signal, $P(f)$ is the false alarm probability, $\sigma_{n}^{2}$ is the noise variance, $\rho$ is the noise uncertainty and $Q^{-1}($.) denotes the inverse Marcum $Q$ function. When the test statistic is compared with the pre-set values of threshold, the energy distribution statistics can be represented as four distinct regions.

\subsection{Anti-Eigen value detector (AVD)}

In AVD the identification edge worth can be determined based on the irregular framework hypothesis. By utilizing this strategy we can accomplish discovery likelihood high and pseudo caution likelihood little without knowing the information of PU signal, commotion force and station data [14]. Consequently commotion uncertainty disadvantage can be overwhelmed by this technique not at all like ED. A run of the mill suspicion in correspondence frameworks is that collector clamor is uncorrelated among time and between different beneficiaries.

Let us assume that $Z$ Eigen values distinguish the binary hypothesis $A 0$ and $A 1$. Therefore, the test statistic for AVD will be the addition of the $\mathrm{Z}$ number of Anti-Eigen values given as

$$
T=\sum_{w=1}^{Z} \widehat{V}_{k}=\sum_{w=1}^{Z} \frac{2 \sqrt{\hat{E}_{w} \hat{E}_{P-w+1}}}{\hat{E}_{w}+\hat{E}_{P-w+1}}
$$

Where $Z$ denotes the rank of the sample covariance matrix which gives the optimum count of small Anti-Eigen value to obtain the test statistic. Eigen esteem put together outputs depending with respect to the biggest Eigen esteem for recognition, similar to the MME and the Generalized Likelihood Ratio Test (GLRT) locator, require a base SNR for discovery to such an extent that the smallest Anti-Eigen value under $A 0$ and $A 1$.

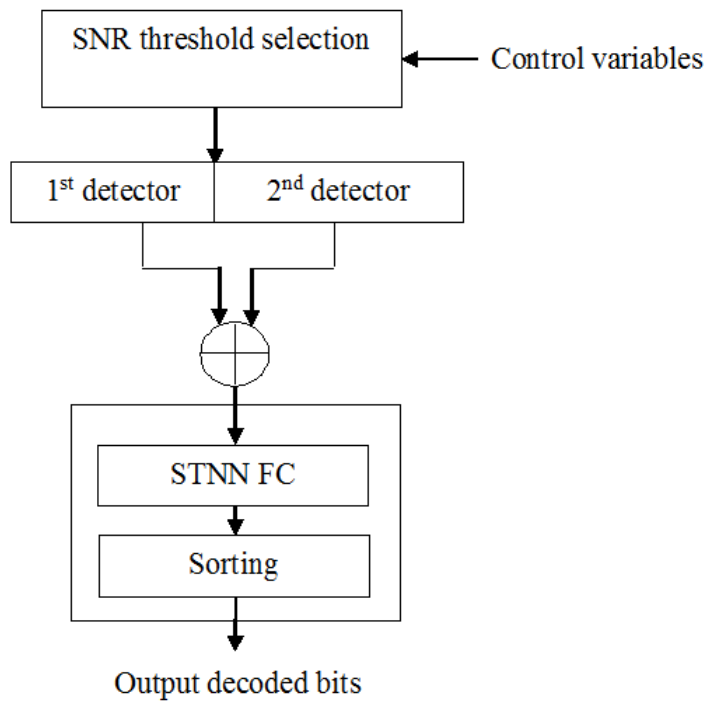

Fig. 2. Methodology implemented. 
Fig. 2 illustrates the functional structure of proposed HBD algorithm with two layer detectors. Depending on the SNR compared to the threshold ED or AVD is selected which is energy detector and Anti-Eigen value detector respectively. Combined results from both the detectors are sent to the fusion center which works on the principle of hard decision based majority fusion rule. Final results were obtained in the form of binary digits, decoded to detect the existence of primary user signal in the sensing channel by the secondary users.

\section{Results and Discussion}

The detection accuracy of the proposed scheme is evaluated in terms of detection parameters which are the miss detection probability, detection probability as well as the false alarm probability. The expressions for the detection probability is given as

$$
P(d)=Q \frac{\left(Q^{-1} P(f)+\sqrt{k / 2}\right)}{S N R_{i}+1}-\sqrt{k / 2}
$$

Where $Q$ (.) is the Marcum $Q$ function, $k$ denotes the sample count and $Q^{-1}($.$) is the$ inverse of $Q$ (.). On the basis of this detection probability the performance of the proposed design can be evaluated with the help of roc curves as shown in Fig. 3. It shows the detection performance of proposed design HBD with respect to different SNR values and it justifies the improved performance at very low SNR signals unlike ED whose detection performance degrades at low SNR values, HBD detects well at very low SNR, upto$10 \mathrm{~dB}$. Although, for a constant false alarm probability, the detection probability degrades as the SNR of the received signal decreases, it detects signal at SNR values lower than $5 \mathrm{~dB}$ more accurately as compared to that of ED. On the other hand AVD performs well at low SNR regions but complexity is more as compared to HBD.

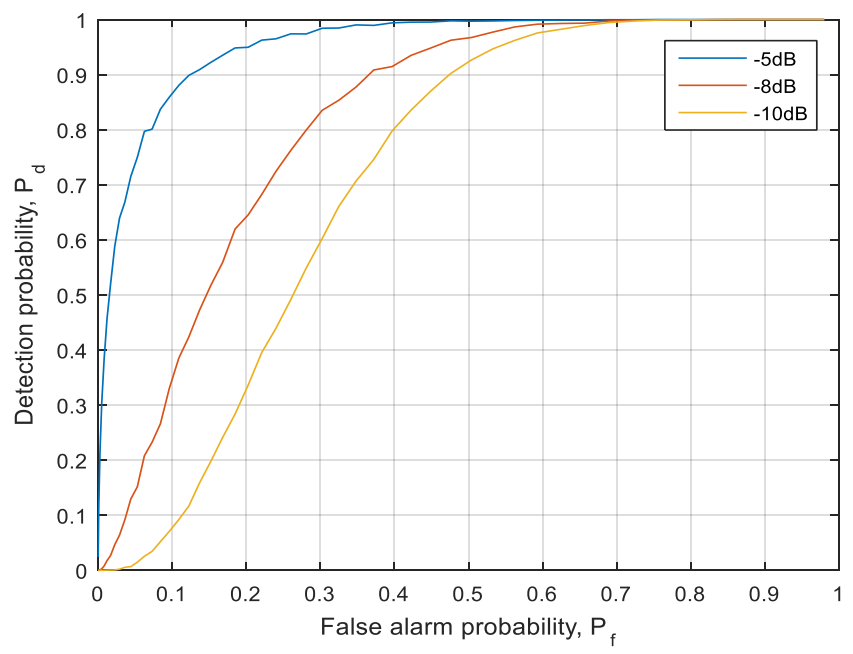

Fig. 3. $\mathrm{P}(\mathrm{d})$ vs $\mathrm{P}(\mathrm{f})$ with $\mathrm{SNR}$ values as $-5 \mathrm{~dB},-8 \mathrm{~dB}$ and $-10 \mathrm{~dB}$. 
Now, the performance of proposed HBD combined detector is compared with the individual existing detectors ED and AVD acting alone with the help of complementary ROC curve as shown in Fig. 4. Here the curve is plotted between the miss detection probability $P(m)$ and false alarm probability $P(f)$, where $P(m)=1-P(d)$. The simulation curve shows that HBD detection performance improves as compared to detection performance of ED and AVD detecting individually. Each technique is analysed theoretically as well as through simulation curves.

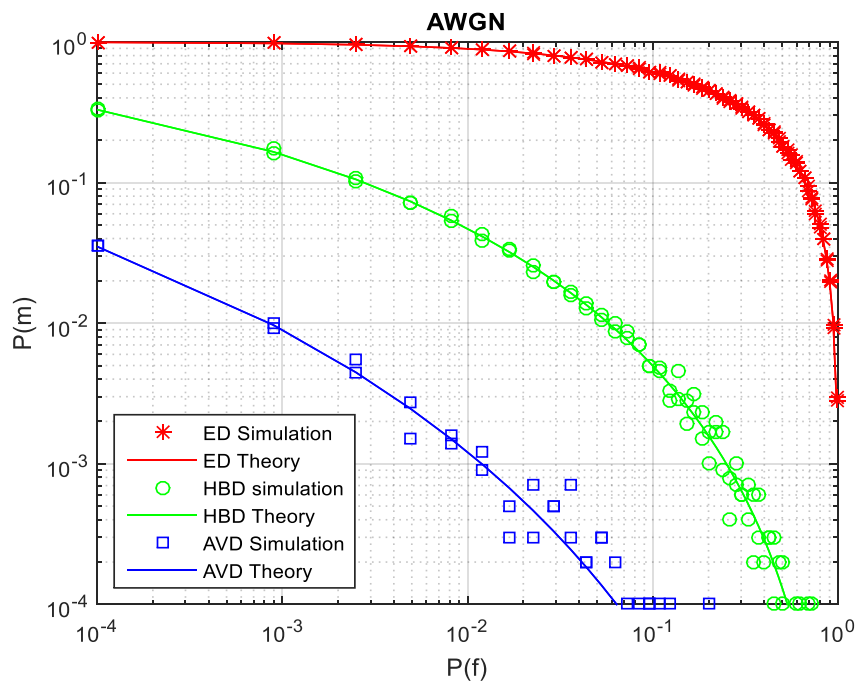

Fig. 4. Performance comparison of HBD with ED and AVD detectors.

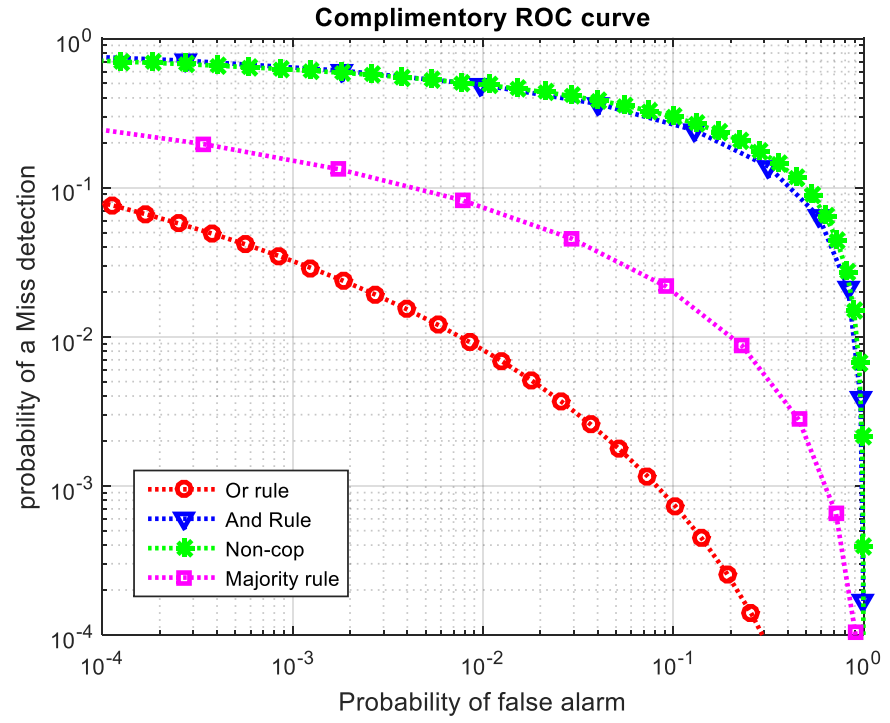

Fig. 5. Performance of HBD for different hard decision fusion rules. 
Now, one of the most challenging issues is to decide the voting rule at the fusion centre. For this purpose a simulation is done using all the three voting rules AND rule, OR rule and majority rule as shown in Fig. 5. Majority rule proves to be the best among the different voting rules to give the better detection accuracy. It also includes the curve for the non- cooperative SS which represents the detection from a single CR only performing local sensing without any cooperation.

\section{Conclusion}

The detection performance of the proposed scheme is analyzed with the help of ROC and CROC curves between the detection probabilities. The paper concludes that the proposed HBD detection performance is increased due to combined detection of two conventional detectors ED and AVD. It combines the advantages of both the detectors, as ED detection is fast as compared to other techniques and implementation is easy and AVD detects well at low SNR regions where ED fails. The advantages of both the individual techniques compensate each other to obtain an enhanced Hybrid Blind Detection based spectrum sensing technique. The other part of the research concluded in this paper is the hard fusion techniques, which depicts that the majority rule performs more accurately as compared to the OR rule and AND rule for a cooperative SS environment.

\section{References}

1. V. Sharma and S. Joshi, Proc. IEEE, 2, 883 (2018). https://doi.org/10.1109/ICCONS.2018.8663089

2. S. Nallagonda, S. D. Roy, and S. Kunda, Cooperative Spectrum Sensing with Censoring of Cognitive Radios in Rayleigh Fading Channel - National Conf. on Communications (NCC) (Kharagpur, India, 2012) pp. 1-5.https://doi.org/10.1109/NCC.2012.6176780

3. R. Viswanathan, Cooperative Spectrum Sensing for Primary User Detection in Cognitive Radio - Fifth Int. Conf. on Sensing Technology (Palmerston North, New Zealand, 2011) pp. 79-84. https://doi.org/10.1109/ICSensT.2011.6137073

4. T. Yucek and H. Arslan, IEEE Communic. Surveys Tutorials 11, 116 (2009). https://doi.org/10.1109/SURV.2009.090109

5. I. F. Akyildiz, W. Y. Lee, and K. R. Chowdhury, Ad Hoc Networks 7, 810 (2009). https://doi.org/10.1016/j.adhoc.2009.02.001

6. A. Satheesh, S. H. Aswini, S. G. Lakshmi, S. Sagar, and K. M. Haresh, Specimen Sensing Techniques A Comparison Between Energy Detector and Cyclostationarity Detector - In Int. Conf. on Central Communication and Computing (ICCC), (Thiruvananthapuram, India, 2013) pp. 388-393. https://doi.org/10.1109/ICCC.2013.6731685

7. M. Emara, H. S. Ali, S. E. A. Khamis, and F. E. A. E. I-Samie, Wireless Personal Commun. 86, 925 (2015). https://doi.org/10.1007/s11277-015-2962-5

8. X. Zhai, Y. Nie, X. Ye, T. Wang, and H. Nie, A Frequency Domain Two-stage Spectrum Sensing Method Based on SVM -IEEE Int. Conf. on Electro Information Technol. (EIT), 2017). https://doi.org/10.1109/EIT.2017.8053339

9. F. Awin, E. Abdel-Raheem and K. Tepe, IEEE Commun. Surveys Tutorials 21, 238 (2019). https://doi.org/10.1109/COMST.2018.2863681

10. S. Haykin, IEEE J. Selected Areas Commun. 25, 201 (2005). https://doi.org/10.1109/JSAC.2004.839380 
11. F. B. S. De Carvalho, W. T. A. Lopes, and M. S. Alencar, Procedia Compt. Sci. 65, 140 (2015). https://doi.org/10.1016/j.procs.2015.09.095

12. D. Kakkar, M. Gupta, A. Khosla, and M. Uddin, Energy Detection Based Spectrum Sensing Analysis of Cooperative Cognitive Radios Under Different Fading Environments, In Cognitive Radio Technology Applications for Wireless and Mobile Ad Hoc Networks (IGI Global, 2013) pp. 50-73. https://doi.org/10.4018/978-1-4666-4221-8.ch003

13. F. F. Digham, M. -S. Alouini, and M. K. Simon, IEEE Trans. Commun. 55, 21 (2007). https://doi.org/10.1109/TCOMM.2006.887483

14. C. Guo, M. Jin, Q. Guo, and Y. Li, IEEE Wireless Commun. Lett. 8, 544 (2018). https://doi.org/10.1109/LWC.2018.2879339 